\title{
VII. APLICABILIDADE DE MÉTODOS ALTERNATIVOS AO USO DE ANIMAIS NO CONTROLE DE QUALIDADE DE BIOLÓGICOS: MODELO DA VACINA CONTRA FEBRE AMARELA.
}

Elaine Cristina Azevedo Navega ${ }^{1}$; Alessandra Santos Almeida ${ }^{1}$; Cristiane Caldeira da Silva²; Octavio Presgrave²; Daniel Silva Guedes ${ }^{1}$; Igor Barbosa da Silva $^{1}$; Isabella Fernandes Delgado ${ }^{3}$; Katherine Antunes de Mattos ${ }^{1}$.

${ }^{1}$ Laboratório de Controle Microbiológico, Dpto. de Controle de Qualidade, Instituto de Tecnologia em Imunobiológicos (Bio-Manguinhos), Fiocruz;

2 Departamento de Farmacologia e Toxicologia Instituto Nacional de Controle de Qualidade em Saúde, Centro Brasileiro para Validação de Métodos Alternativos, Fiocruz;

3 Departamento de Farmacologia e Toxicologia, Instituto Nacional de Controle da Qualidade em Saúde, Vice Diretoria de Pesquisa e Ensino do Instituto Nacional de Controle de Qualidade em Saúde, Fiocruz.

INTRODUÇÃo A necessidade de alternativas ao uso de animais no teste de pirogênio é guiada pelo principio dos 3R's, culminando na aceitação pela Farmacopeia Europeia (F.E.) do método alternativo in vitro, o Teste de Ativação de Monócitos (MAT, 2010). Respeitando esta premissa, o MAT utiliza como matriz, fontes de monócitos humanos, sendo considerado um método promissor e eficiente, por excluir riscos inerentes à extrapolação inter-espécies, detectar amplo espectro de pirogênios e suprir limitações dos testes atuais, o Teste de Endotoxina Bacteriana (LAL) e o Teste de Pirogênio em coelhos. Apesar das vantagens abordadas, alguns obstáculos técnicocientíficos-regulatórios devem ser transpostos para a implantação efetiva do MAT na rotina industrial, em especial, de produtos biológicos.

OBJETIVO Neste contexto, a vacina contra febre amarela (VFA) foi selecionada como primeiro modelo de aplicabilidade do MAT em vacinas por: (1) ser carro-chefe do portfólio de Bio-Manguinhos, (2) processo de produção complexo, com fatores críticos de interferência como uso de vírus vivo e resíduos de componentes de processos (ovos embrionados), (3) potencial investigativo de outras fontes pirogênicas não detectadas pelo método descrito na monografia da vacina.

METODOLOGIA Os métodos farmacopeicos descrito para MAT utilizando IL-1/sangue criopreservado, IL-6/ sangue fresco e proposta de método para rotina industrial, IL-6/ sangue criopreservado, foram validados conforme requisitos da F.E. A verificação produto-específica foi realizada com a VFA/10 doses e fatores interferentes 
avaliados através da máxima diluição válida. Após determinação da diluição, o método quantitativo foi aplicado aos lotes vacinais, com interpretação entre os sistemas de leitura e matrizes do MAT e LAL.

RESULTADOS Os dados mostram reprodutibilidade do teste utilizando diferentes doadores e medidores de pirogenicidade (IL-6/IL-1) frente ao estímulo da endotoxina. Após verificação da aplicabilidade da VFA ao MAT com diluição 1:10, demonstramos que os sistemas utilizando sangue criopreservado (IL-1/IL-6) respeitam os limites de recuperação da endotoxina e se mostraram mais adequados do que o sistema IL-6/sangue fresco. Os resultados quantitativos da resposta pirogênica no produto determinada pelo MAT correspondem àqueles observados pelo LAL.

CONCLUSÃo Os dados demonstram pela primeira vez a aplicabilidade do MAT em vacinas, sendo o modelo VFA compatível com a utilização de sangue criopreservado, acessível à rotina industrial. Este trabalho reflete o compromisso da maior produtora nacional de vacinas com a saúde pública e bem-estar animal, obediência das diretrizes internacionais, cuidado com o controle da qualidade e contribuição cientifica para a expansão da tecnologia e do conhecimento na avaliação de biológicos com end-points humanos.

PALAVRAS-CHAVE métodos alternativos, pirogênio, teste de ativação de monócitos, biológicos, febre amarela. 